DE

M E D I C I N A

T R O P I C A L

$\mathrm{DE}$

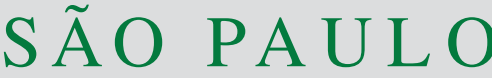

JOURNAL OF THE SÃO PAULO INSTITUTE OF TROPICAL MEDICINE

${ }^{1}$ Universidade de São Paulo, Hospital das Clínicas, Divisão Dermatologia, Laboratório de Dermatologia e Imunodeficiências, São Paulo, São Paulo, Brazil

${ }^{2}$ Universidade de São Paulo, Instituto de Medicina Tropical de São Paulo, São Paulo, São Paulo, Brazil

${ }^{3}$ Instituto de Infectologia Emílio Ribas, São Paulo, São Paulo, Brazil

${ }^{4}$ Universidade de São Paulo, Faculdade de Medicina, Departamento de Medicina Preventiva, São Paulo, São Paulo, Brazil

5Universidade de São Paulo, Escola de Medicina de Ribeirão Preto, Departamento de Medicina Interna, Ribeirão Preto, São Paulo, Brazil

Correspondence to: Marilia Ladeira de Araújo

Universidade de São Paulo, Instituto de Medicina Tropical de São Paulo,

Av. Dr. Eneas de Carvalho Aguiar, 470, Cerqueira Cesar, CEP 05403- 903,

São Paulo, SP, Brazil

Tel: +55 11 3061-7499

Fax: $+55113081-7190$

E-mail: ladeira.araujo @gmail.com

Received: 26 September 2017

Accepted: 19 February 2018

\section{Is the telomere length associated with neurocognitive disabilities in HIV-1-infected subjects?}

\author{
Marilia Ladeira de Araújo1,2, Wellington Duarte', Augusto César Penalva \\ de Oliveira ${ }^{3}$, Maria Rita Polo Gascón ${ }^{3}$, Luiz Augusto Marcondes Fonseca ${ }^{4}$, \\ Raquel de Melo Alves Paiva ${ }^{5}$, Bárbara Santana ${ }^{5}$, Rodrigo Tocantins Calado ${ }^{5}$, \\ Jorge Casseb ${ }^{1,2}$
}

\section{ABSTRACT}

Objective: We evaluated the association between cognitive deficits and leukocyte telomere length (LTL) in HIV-1-infected individuals. Design: 73 HIV-1-infected patients undergoing neuropsychological evaluation and 91 healthy controls were included in this study. Fifteen HIV-1 positive patients did not have cognitive disorders whereas 26 had asymptomatic neurocognitive disorder (ANI), 13 presented mild to moderate neurocognitive disorder (MND), and 10 had HIV-associated dementia (HAD). Methods: DNA from the peripheral blood of HIV-1-infected patients was used for measurement of telomere length by real-time PCR. HIV-1 viral load was determined in blood. Results: LTL decreased with age in healthy controls ( $\mathrm{p}=0.0001)$. Regardless of the HIV status, age-matched LTL from HIV patients, including those with ANI and MND, were shortened in comparison to the healthy control group ( $\mathrm{p}=0.0073$ ); however, no association was found among the HIV-1-infected individuals with cognitive deficits $(\mathrm{p}=0.01)$. In addition, no gender-related association with LTL was observed $(\mathrm{p}=0.80)$, smoking, physical exercise, and plasma viral load were not correlated to telomere length ( $\mathrm{p}=0.66)$. Conclusions: We concluded that leukocyte telomere length may not be a marker of cellular senescence in individuals with HIV infection and neurocognitive disorders.

KEYWORDS: Cell aging. Neurocognitive disorders. Real-time polymerase chain reaction. AIDS. Dementia complex.

\section{INTRODUCTION}

Human immunodeficiency virus type 1 (HIV-1) infection remains a serious public health problem ${ }^{1}$. There are more than 40 million HIV-infected patients worldwide, most of them showing some degree of cognitive impairment. The use of highly active antiretroviral therapy (HAART) has changed the natural history of the infection ${ }^{2}$, increasing patients' life expectancy but heightening the chance of damage to the central nervous system (CNS).

The mechanism of CNS lesions involves virus entry into the CNS during the early course of infection through the disruption of the blood-brain barrier (BBB). This event results from the neurotoxic activity of several HIV-1 proteins, including gp120, tat, vpr, and others ${ }^{3}$. In addition, there are alterations both in the CNS homeostasis, involving the metabolic integrity of the $\mathrm{BBB}$, and in the metabolism of astrocytes, microglia, resident macrophages and perivascular space ${ }^{4}$.

Due to the processes described above, neurocognitive disorders may ensue. 
According to the classification revised by the working group assembled by Frascatti in 2007, neurocognitive disorders associated with HIV (HAND) are classified as asymptomatic neurocognitive impairment (ANI), mild cognitive disorder (MND), and HIV-associated dementia $(\mathrm{HAD})^{5}$. A significant increase in the incidence of cognitive dysfunction in HIV-infected patients has occurred in the last years ${ }^{6}$.

The cognitive decline is associated with age and may be accelerated in patients with HIV. This fact may be related to telomere shortening ${ }^{7,8}$. Telomeres are nucleoproteins consisting of several thousand DNA repeats of TTAGGG in association with a protein complex at the end of linear chromosomes ${ }^{9}$. Telomeres act to protect the chromosome ends from recognition as damaged or infectious DNA ${ }^{10,11}$.

Telomeric DNA shortens during each cell replication and, when critically short, telomere length (TL) can trigger the cell to enter replicative senescence or apoptosis and, in cells that continue to divide, to chromosomal abnormality ${ }^{11-15}$. The etiology of immunosenescence is multifactorial and reflects exposure to external pathogens, persistent viral infections, obesity, physical and psychological stress, and thymic involution, among other events throughout an individual's life ${ }^{16}$.

HIV infection can lead to chronic immune activation, oxidative stress, and inflammation ${ }^{17}$ and is also correlated with shortened telomeres ${ }^{18}$ in vivo ${ }^{19,20}$ and inhibition of telomerase in vitro ${ }^{21}$. Chronic immune activation due to persistence of circulating HIV virions may play a key role in the senescent pathway. Activated cells undergo clonal expansion in response to viral persistence, resulting in differentiation and accumulation of nonfunctional senescent cells ${ }^{22}$.

Comparing TL in HIV-infected and HIV-uninfected individuals may be an important step toward understanding why HIV-infected individuals have a higher incidence of age-related diseases ${ }^{23}$. Thus, the objective of this work was to evaluate the impact of HIV infection in the immunosenescence of leukocytes, in patients with neurocognitive disturbance, in addition to providing possible biomarkers to this pathological code, independent of neurocognitive compromise. Some risk factors, such as smoking, physical activity and other factors were also evaluated.

\section{MATERIAL AND METHODS}

Mean TL was measured by qPCR based on a modification of the method described by Cawthon in 2002, as previously described ${ }^{24-26}$. To ensure the quality of our data, all samples $(100 \mathrm{ng})$ were loaded in agarose gel $1 \%$ to check the DNA integrity. Samples that were not degraded were submitted to qPCR. Basically, qPCR was conducted in triplicate and reactions included: genomic DNA (1.6 ng), 2x RotorGene SYBR Green, PCR Master Mix (Qiagen, Hilden, Germany), RNase-free water (Qiagen, Germany), primer Tel Forward (300 nM) (CGGTTTGTTTGGGTTTGGG TTTGGGTTTGGGTTTGGGTT) and Tel Reverse (300 nM) (GGCTTGCCTTACCCTTACCCTTACCCTTACCC TTACCCT) or primer single gene forward (36B4 F-300 nM) (CAGCAAGTGGGAAGGTGTAATCC) and single gene reverse (36B 4 R-500 $\mathrm{nM}$ ) (CCCATTCTATCATCAACGGGTACAA), in a $24 \mu \mathrm{L}$ final reaction. All qPCR reactions were prepared on a QIAgility automated pipettor (Qiagen, California, USA) and amplification was conducted in the Rotor-Gene Q real-time PCR cycler as follow: $5 \mathrm{~min}$ at $95{ }^{\circ} \mathrm{C}, 25$ cycles (telomere reaction) and 35 cycles (single gene reaction) of $7 \mathrm{~s}$ at $98{ }^{\circ} \mathrm{C}$ and $10 \mathrm{~s}$ at $60{ }^{\circ} \mathrm{C}$ (telomere) or $10 \mathrm{~s}$ at $58{ }^{\circ} \mathrm{C}$ (single gene). The TL for each sample was determined using the telomere to single copy gene ratio ( $\mathrm{T} / \mathrm{S}$ ratio) by calculating the $\Delta \mathrm{Ct}[\mathrm{Ct}($ telomere $) / \mathrm{Ct}($ single gene $)]$. The $\mathrm{T} / \mathrm{S}$ ratio for each sample (x) was normalized to the mean $\mathrm{T} / \mathrm{S}$ ratio of reference sample $[2-(\Delta \mathrm{Ctx}-\Delta \mathrm{Ctr})=2-\Delta \Delta \mathrm{Ct}]$, which was also used for the standard curve, both as a reference sample and as a validation sample. In every run, two reference samples were included to validate each reaction. The experiment was considered acceptable if control sample $\mathrm{T} / \mathrm{S}$ ratio ranged within the $95 \%$ variation interval (0.95-1.05). The correlation between TL measurements of an independent cohort $(n=76)$ by qPCR and Southern blot was used to convert $\mathrm{T} / \mathrm{S}$ ratio values in kilobases. The linear regression equation used was: telomere length $(\mathrm{kb})=4.330 \mathrm{x}+5.07\left(\mathrm{R}^{2}=0.55 ; \mathrm{p}<0.0001\right)$, where $\mathrm{x}$ corresponds to the $\mathrm{T} / \mathrm{S}$ ratio value.

\section{Ethics}

The Scientific Committee and the Research Ethics Committee, both from the Institute of Infectious Diseases Emilio Ribas, São Paulo, Brazil, approved this study (034/11). Participant samples and other research data were collected within one year from the neuropsychological analysis of the participants of this study. Detailed history of each individual, including information as an indicator of physical activity practice and its relationship with the quality of life of young adults (through the IPAQ questionnaire), time of HIV infection, whether the participant is a smoker or not; Data on CD4 + T-cell lymphocyte counts and nadir of CD4+ counts were also obtained. All participants signed a written informed consent form.

The application of neuropsychological tests was 
performed by a team of highly trained neuropsychologists at the Institute of Infectious Diseases Emilio Ribas. The data collection from patients included in this study comprised five sections: 1) socio-demographic survey; 2) medical history and survey of functional status; 3 ) questionnaire of subjective neurological symptoms; 4) complete neurological evaluation; 5) neuropsychological screening test (International HIV Dementia Scale) followed by neuropsychological tests.

In addition to establishing the neuropsychological profile of patients, we evaluated the indicator of physical activity practice and its relationship with the quality of life of young adults of physical activity using the International Physical Activity Questionnaire (IPAC) (Table 1), developed by the World Health Organization (WHO, 1998).

A Neurology American Academy 2007 criterion was used to characterize patient groups: asymptomatic neurocognitive disorder (ANI), MND and $\mathrm{HAD}^{5}$ Neuropsychological tests evaluated the following criteria: attention/concentration, information processing speed, executive function, reasoning, abstraction, memory/ learning, visuospatial capacity and operational motor.

Exclusion criteria were individuals presenting depression during neuropsychological tests, individuals using abusive and psychotropic drugs, pregnant women, and individuals with serologically positive results, such as toxoplasmosis, syphilis, hepatitis B or C, and other diseases that may result in some form of neurological disorder. Inclusion criteria were patients of both genders, from 35 to 60 years of age, who met the criteria according to the neuropsychological evaluation.

Parametric tests were used for normally distributed variables, Mann-Whitney test for nonparametric distributions, and the Spearman and Pearson's $r$ tests for analysis of correlation, where appropriate. One-way ANOVA - Dunnett tests were used to detect differences of telomere length, as a continuous variable. For the elaboration of graphs, we employed Graph Pad Software 5.0 (La Jolla, CA, US). Statistical significance level was set at $\mathrm{p} \leq 0.05$.

\section{RESULTS}

From the 73 patients included in this study and submitted to the neurological evaluation, 15 did not have a cognitive impairment whereas 49 were cognitively impaired and classified under one of the three categories of asymptomatic neurocognitive disorder (ANI), mild to moderate neurocognitive disorder (MND), and HIVassociated dementia (HAD). 91 HIV-uninfected healthy individuals made up the control group for this study.

The demographic and clinical characteristics of the patients are shown on Table 1. Only three patients had a detectable viral load in their cerebrospinal fluid. Of the 73 HIV-1-infected patients, 20 (31.25\%) were female and 44 $(68.75 \%)$ were male. The LTL was performed by qPCR for

Table 1 - Demographical, laboratorial and clinical characteristics of the participants

\begin{tabular}{|c|c|c|c|c|c|c|}
\hline Variable & $\begin{array}{c}\text { Control } \\
\text { n. (\%) }\end{array}$ & $\begin{array}{c}\text { Normal } \\
\text { n. (\%) }\end{array}$ & $\begin{array}{c}\text { ANI } \\
\text { n. (\%) }\end{array}$ & $\begin{array}{l}\text { MND } \\
\text { n. (\%) }\end{array}$ & $\begin{array}{l}\text { HAD } \\
\text { n. }(\%)\end{array}$ & $p$ value \\
\hline Total & 91 & 15 & 26 & 13 & 10 & \\
\hline 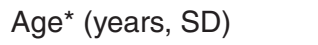 & $46.79(7.36)$ & $46.73(6.37)$ & $49.31(5.64)$ & $52.0(6.94)$ & $49.7(6.75)$ & $<0.0001$ \\
\hline $\begin{array}{l}\text { Gender } \\
\text { Male } \\
\text { Female }\end{array}$ & $\begin{array}{l}55 / 91 \\
36 / 91\end{array}$ & $\begin{array}{l}11 / 15 \\
04 / 15\end{array}$ & $\begin{array}{l}18 / 26 \\
08 / 26\end{array}$ & $\begin{array}{l}10 / 13 \\
03 / 13\end{array}$ & $\begin{array}{c}05 / 10 \\
0510\end{array}$ & \\
\hline Education* (years) & $15.58(2.58)$ & $15.67(5.12)$ & $11.50(3.03)$ & 11.46 (3.07) & $8.00(3.85)$ & $<0.0001$ \\
\hline Infection * time HIV & N.A. & $11.80(7.72)$ & $9.80(7.12)$ & $10.31(4.59)$ & $15.10(3.24)$ & 0.0813 \\
\hline Current Smoker (\%) & 0 & 26.6 & 3.84 & 15.38 & 0 & \\
\hline Physical* activity $^{*}$ & $3100(2100)$ & 2020 (2133) & $2400(3000)$ & 2150 (2312) & $1500(1300)$ & 0.0850 \\
\hline CPE rank* & N.A. & $5.13(3.22)$ & $7.00(2.54)$ & $5.23(2.04)$ & $7.40(1.35)$ & 0.0308 \\
\hline HIV RNA plasma & N.A. & 23 & 175 & 0 & 17 & \\
\hline T CD4+ cells & N.A. & $753(259)$ & $650(261)$ & $677(269)$ & $463(169)$ & 0.0156 \\
\hline Telomere Length (T/S) & $0.688(0.229)$ & $0.574(0.210)$ & $0.483(0.168)$ & $0.477(0.219)$ & $0.473(0.058)$ & $<0.0001$ \\
\hline
\end{tabular}

Note: Groups classified as HIV negative controls; HIV-infected patients without neurocognitive impairments; ANI: Asymptomatic neurocognitive impairment; MND: Associated mild neurocognitive disorder; HAD: HIV-associated dementia. * represents the values of mean and standard deviation. Physical activity score is divided into: inactive: 0 to 500MET-min/week; modestly active: 600MET to 2999 MET-min / week; active: at least 3000MET-min/week. CPE rank: effectiveness score of antiretroviral drugs penetration in the central nervous system. HIV RNA Blood and CSF means detectable viral load (\%). N.A: not applicable 
the HIV-infected positive patients and for the 91 healthy controls.

Loss of TL with aging was observed for the control group ( $p=0.0001)$. HIV-1-infected patients, including those both with and without neurocognitive disorder presented shorter age-matched telomere length in comparison to healthy individuals $(p=0.0073)$. A statistically significant difference was found among HIV-infected patients: those without neurocognitive disorder had longer telomere lengths than those with cognitive impairment taking as a whole $(p=0.01)$ (Figure 1). However, no significant difference in telomere length was found between HIVpositive individuals without neurocognitive disorders and other groups when the comparison was made with each individual group (ANI, MND, HAD, respectively) $(p=0.14$; $p=0.67 ; p=0.42$ ), suggesting that this characteristic alone is not sufficient to differentiate the levels of neurocognitive disorders in HIV-positive patients.

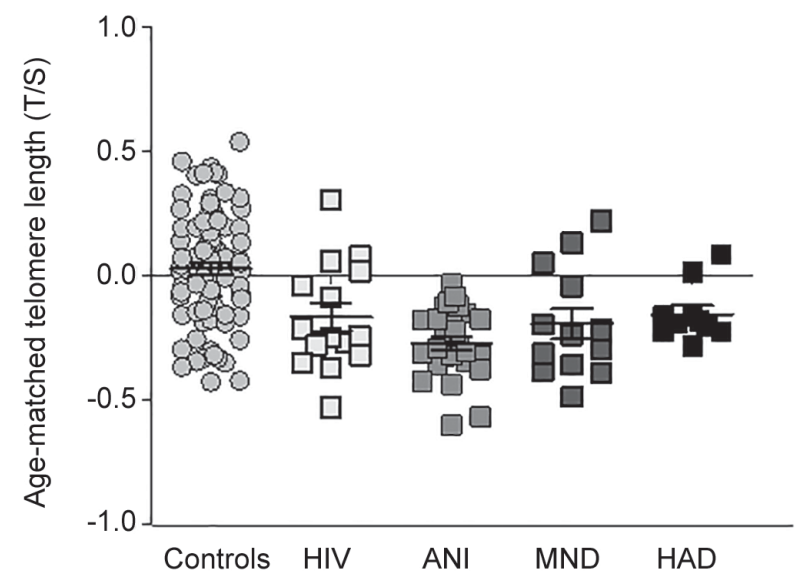

Figure 1 - Telomere length from HIV patients and healthy controls according to their respective performances on neuropsychological tests

Smoking was not associated with telomere length among HIV-positive individuals $(p=0.97)$, and so was physical activity $(p=0.11$ ) (Table 1$)$. Other variables such as plasma viral load, time of infection, and CD+ T lymphocyte count were also not associated with TL $(p=0.66 ; p=0.09 ; p=0.84$, respectively). Additionally, no difference on LTL was found between males and females $(p=0.80)$.

\section{DISCUSSION}

Some studies suggest that short telomeres cause an increase in neurocognitive impairment, which may lead to a risk of dementia in non-HIV infected populations ${ }^{27}$ and may be a marker for atypical cognitive decline related to age ${ }^{28,29}$, while another study pointed out that telomere length does not seem to provide a tool to predict cognitive decline ${ }^{30}$. Currently, there is an increasing incidence of all degrees of neurocognitive impairment in HIV-1-infected patients. Telomere length has been proposed as a possible marker for such neurocognitive alterations. In our study, we did not find an association between leukocytes, telomere lengths and age of patients who were not cognitively impaired. A limitation of our study is the small number of patients with HAD, probably due to the decrease in the incidence of the disorder after the beginning of HAART use in large scale. Previous studies have linked the shortening of telomeres with increasing neurocognitive changes, suggesting that it might be a marker ${ }^{27,28,31}$ and possibly involved in the accelerated cognitive aging of HIV-1-infected individuals.

Consistent with our findings, a previous study that searched for an association of telomere length with neurocognitive impairments in HIV-positive women did not find any was negative ${ }^{32}$. In contrast, another study suggested that telomere length might be a risk factor for the increasing susceptibility to neurocognitive decline in HIV-1- infected individuals ${ }^{31}$.

Furthermore, we examined whether some individual practices, harmful or beneficial, could influence telomere length. We could not find an association between tobacco smoking and LTL. Similarly, physical activity was also not associated with telomere length, contrary to the findings of another study ${ }^{29}$. Some studies suggested that telomere length is longer in women ${ }^{33,34}$, but this remains a controversial issue. We could not find a statistically significant difference in telomere length between genders in the current study.

As expected, older age was associated with telomere length shortening. Other studies have reached similar results, independently of the HIV status ${ }^{3,35}$. There was a significant difference in the telomere length in leukocytes between HIV-infected individuals compared to our HIVnegative healthy controls ${ }^{36,37}$. Similarly, a study showed that CD8+ T cells from HIV-infected individuals had shorter telomeres than the same cells from HIV-negative subjects, reflecting the impact of viral infection on the senescence of that cellular compartment. The same study did not find the same abnormalities on $\mathrm{CD} 4+\mathrm{T}$ cells ${ }^{38}$. We have not performed a study using $\mathrm{T}$ cell subpopulations to test this hypothesis. We suggest that the investigation of lymphocytes should be performed with purified cells instead of total leukocytes.

In conclusion, telomere shortening was not associated with neurocognitive impairment in our HIV-infected patients and did not act as a cellular aging biomarker in this population. Further studies are needed to better understand the mechanisms of HIV infection and senescence in patients with different degrees of neurocognitive disorders. 


\section{ACKNOWLEDGMENTS}

We thank all the people who collaborated to carry out this research.

\section{CONFLICT OF INTERESTS}

All authors declared no conflict of interest.

\section{AUTHORS' CONTRIBUTIONS}

Wellington Duarte did the scheduling and collection of patients' material; Augusto Cesar Penalva de Oliveira gave the co-guidance for the execution of this research; the neuropsychologist Maria Rita Polo Gascón applied the battery of neuropsychological tests to patients; Raquel Paiva, Bárbara Santana and Rodrigo Tocantins Calado collaborated to carry out the analyzes.

\section{REFERENCES}

1. Centers for Disease Control and Prevention. HIV surveillance reports: diagnoses of HIV infection in the United States and dependent areas, 2011. [cited 2017 Sept 12]. Available from: https://www.cdc.gov/hiv/pdf/statistics_2011_hiv_ surveillance_report_vol_23.pdf

2. Cañizares S, Cherner M, Ellis R. HIV and aging: effects on the central nervous system. Semin Neurol. 2014;34:27-34.

3. Barrett EL, Richardson DS. Sex differences in telomeres and lifespan. Aging Cell. 2011;10:913-21

4. Rao VR, Ruiz AP, Prasad VR. Viral and cellular factors underlying neuropathogenesis in HIV associated neurocognitive disorders (HAND). AIDS Res Ther. 2014;11:13.

5. Antinori A, Arendt G, Becker JT, Brew BJ, Byrd DA, Cherner M, et al. Updated research nosology for HIVassociated neurocognitive disorders. Neurology. 2007;69: 1789-99.

6. Haziot ME, Barbosa Junior SP, Vidal JE, Oliveira FT, Oliveira AC. Neuroimaging of HIV associated neurocognitive disorders. Dement Neuropsychol. 2015;9:380-4.

7. Honig LS, Schupf N, Lee JH, Tang MX, Mayeux R. Shorter telomeres are associated with mortality in those with APOE $€ 4$ and dementia. Ann Neurol. 2006;60:181-7.

8. Maj M, Janssen R, Starace F, Zaudig M, Satz P, Sughondhabirom B, et al. WHO Neuropsychiatric AIDS study, cross-sectional phase I. Study design and psychiatric findings. Arch Gen Psychiatry. 1994;51:39-49.

9. Greider CW. Telomere length regulation. Annu Rev Biochem. 1996;65:337-65.

10. Harley CB, Futcher AB, Greider CW. Telomeres shorten during ageing of human fibroblasts. Nature. 1990;345:458-60.
11. Calado RT, Young NS. Telomere diseases. N Engl J Med. 2009;361:2353-65.

12. Streffer C. Strong association between cancer and genomic instability. Radiat Environ Biophys. 2009;49:125-31.

13. Raynaud CM, Jang SJ, Nuciforo P, Lantuejoul S, Brambilla E, Mounier N, et al. Telomere shortening is correlated with the DNA damage response and telomeric protein down-regulation in colorectal preneoplastic lesions. Ann Oncol. 2008;19:187581

14. Paiva RM, Calado RT. Telomere dysfunction and hematologic disorders. Prog Mol Biol Transl Sci. 2014;125:133-57.

15. Feldser DM, Hackett JA, Greider CW. Telomere dysfunction and the initiation of genome instability. Nat Rev Cancer. 2003;3:623-7.

16. de Araújo AL, Silva LC, Fernandes JR, Benard G. Preventing or reversing immunosenescence: can exercise be an immunotherapy? Immunotherapy. 2013;5:879-93.

17. Pace GW, Leaf CD. The role of oxidative stress in HIV disease. Free Radic Biol Med. 1995;19:523-8.

18. Oeseburg H, de Boer RA, van Gilst WH, van der Harst P. Telomere biology in healthy aging and disease. Pflügers Arch. 2010;459:259-68.

19. Palmer S, Perry J, Kipling D, Ashworth A. A gene spans the pseudoautosomal boundary in mice. Proc Natl Acad Sci U S A. $1997 ; 94: 12030-5$

20. Pathai S, Bajillan H, Landay AL, High KP. Is HIV a model of accelerated or accentuated aging? J Gerontol A Biol Sci Med Sci. 2013;69:833-42.

21. Diniz LF, Cruz MF, Torres VM, Cosenza RM. O teste de aprendizagem auditivo-verbal de Rey: normas para uma população brasileira. Rev Bras Neurol. 2000;36:79-83.

22. Desai S, Landay A. Early immune senescence in HIV disease. Curr HIV/AIDS Rep. 2010;7:4-10.

23. Auld E, Lin J, Chang E, Byanyima P, Ayakaka I, Musisi E, et al. HIV infection is associated with shortened telomere length in Ugandans with suspected tuberculosis. PLoS One. 2016;11:e0163153.

24. Gutierrez-Rodrigues F, Santana-Lemos BA, Scheucher PS, AlvesPaiva RM, Calado R. Direct comparison of flow-FISH and qPCR as diagnostic tests for telomere length measurement in humans. PLoS One. 2014;9:e113747.

25. Scheinberg P, Cooper JN, Sloand EM, Wu CO, Calado RT, Young NS. Association of telomere length of peripheral blood leukocytes with hematopoietic relapse, malignant transformation, and survival in severe aplastic anemia. JAMA. 2010;304:1358-64.

26. Calado RT, Cooper JN, Padilla-Nash HM, Sloand EM, Wu CO, Scheinberg P, et al. Short telomeres result in chromosomal instability in hematopoietic cells and precede malignant evolution in human aplastic anemia. Leukemia. 2011;26:700-7 
27. von Zglinicki T, Serra V, Lorenz M, Saretzki G, LenzenGroßimlighaus R, Geßner R, et al. Short telomeres in patients with vascular dementia: an indicator of low antioxidative capacity and a possible risk factor?. Lab Invest. 2000;80:173947.

28. Martin-Ruiz C, Dickinson HO, Keys B, Rowan E, Kenny RA, von Zglinicki T. Telomere length predicts poststroke mortality, dementia, and cognitive decline. Ann Neurol. 2006;60:174-80.

29. Yaffe K, Lindquist K, Kluse M, Cawthon R, Harris T, Hsueh WC, et al. Telomere length and cognitive function in communitydwelling elders: findings from the Health ABC Study. Neurobiol Aging. 2011;32:2055-60.

30. Devore EE, Prescott J, De Vivo I, Grodstein F. Relative telomere length and cognitive decline in the Nurses' Health Study. Neurosci Lett. 2011;492:15-8.

31. Malan-Müller S, Hemmings SM, Spies G, Kidd M, FennemaNotestine C, Seedat S. Shorter telomere length - a potential susceptibility factor for HIV-associated neurocognitive impairments in South African women. PLoS One. 2013;8:e58351.

32. Giesbrecht CJ, Thornton AE, Hall-Patch C, Maan EJ, Côté HC, Money DM, et al. Select neurocognitive impairment in HIVinfected women: associations with HIV viral load, hepatitis C virus, and depression, but not leukocyte telomere length. PLoS One. 2014;9:e89556.
33. Bekaert S, De Meyer T, Rietzschel ER, De Buyzere ML, De Bacquer D, Langlois $\mathrm{M}$, et al. Telomere length and cardiovascular risk factors in a middle-aged population free of overt cardiovascular disease. Aging Cell. 2007;6:639-47.

34. Fitzpatrick AL, Kronmal RA, Gardner JP, Psaty BM, Jenny NS, Tracy RP, et al. Leukocyte telomere length and cardiovascular disease in the cardiovascular health study. Am J Epidemiol. 2007;165:14-21.

35. Pathai S, Lawn SD, Gilbert CE, McGuinness D, McGlynn L, Weiss HA, et al. Accelerated biological ageing in HIV-infected individuals in South Africa. AIDS. 2013;27:2375-84.

36. Palmer LD, Weng N, Levine BL, June CH, Lane HC, Hodes RJ. Telomere length, telomerase activity, and replicative potential in HIV infection: analysis of CD4+ and CD8+T cells from HIVdiscordant monozygotic twins. J Exp Med. 1997;185:1381-6.

37. Zanet DL, Thorne A, Singer J, Maan EJ, Sattha B, Le Campion A, et al. Association between short leukocyte telomere length and HIV infection in a cohort study: No evidence of a relationship with antiretroviral therapy. Clin Infect Dis. 2014;58:1322-32.

38. Liu JC, Leung JM, Ngan DA, Nashta NF, Guillemi S, Harris M, et al. Absolute leukocyte telomere length in HIV-infected and uninfected individuals: evidence of accelerated cell senescence in HIV-associated chronic obstructive pulmonary disease. PLoS One. 2015;10:e0124426. 